\title{
Systematic review and meta-analysis of ultrasonic elastography in the diagnosis of benign and malignant thyroid nodules
}

\author{
Ying Zhou ${ }^{1}$, Huali Chen ${ }^{1}$, Jialin Qiang', Dandan Wang ${ }^{2}$ \\ ${ }^{1}$ Ultrasonography Department, Zhejiang Quhua Hospital, Quzhou, China; ${ }^{2}$ Ultrasonography Department, The Second Affiliated Hospital of \\ Zhejiang Chinese Medicine University, Hangzhou, China \\ Contributions: (I) Conception and design: Y Zhou, D Wang; (II) Administrative support: H Chen; (III) Provision of study materials or patients: \\ Y Zhou, J Qiang, D Wang; (IV) Collection and assembly of data: All authors; (V) Data analysis and interpretation: Y Zhou, H Chen, J Qiang; (VI) \\ Manuscript writing: All authors; (VII) Final approval of manuscript: All authors. \\ Correspondence to: Dandan Wang. Ultrasonography Department, The Second Affiliated Hospital of Zhejiang Chinese Medicine University, No. 318 \\ Chaowang Road, Gongshu District, Hangzhou, China. Email: n1958c@163.com.
}

Background: In recent years, ultrasound elastography (USE) has been the main diagnostic technique for benign and malignant thyroid nodules. However, it cannot display the blood flow signals of nodules with smaller diameters clearly, which decreases its diagnostic accuracy.

Methods: Chinese and English databases were searched using "ultrasonic elastography", "benign and malignant thyroid nodules", and "diagnose" as the search terms. RevMan 5.3 software was used for metaanalysis.

Results: A total of 11 randomized controlled trials (RCTs) were included. Eight articles analyzed clinical symptoms, and the heterogeneity test results were $\mathrm{Chi}^{2}=7.46, \mathrm{df}=7, \mathrm{I}^{2}=6 \%$, and $\mathrm{P}=0.38$; and $\mathrm{Z}=11.44$, OR $=14.58,95 \%$ confidential interval (CI): 9.21-23.07, and $\mathrm{P}<0.01$. The diagnostic efficacy of USE was analyzed in 8 articles, and the heterogeneity test results were $\mathrm{Chi}^{2}=3.03, \mathrm{df}=7, \mathrm{I}^{2}=0 \%$, and $\mathrm{P}=0.88$; and $\mathrm{Z}=6.33$, $\mathrm{OR}=7.47,95 \% \mathrm{CI}: 4.01-13.93$, and $\mathrm{P}<0.01$. The pathological diagnosis results of randomized controlled trials were analyzed in 10 literatures, and the heterogeneity test results were $\mathrm{Tau}^{2}=0.78, \mathrm{Chi}^{2}=20.97, \mathrm{df}=9$, $\mathrm{I}^{2}=57 \%$, and $\mathrm{P}=0.01$; and $\mathrm{Z}=7.27, \mathrm{OR}=14.67,95 \% \mathrm{CI}: 7.11-30.27$, and $\mathrm{P}<0.01$. After meta-analysis, the combined sensitivity was $72.26 \%$ (95\% CI: $0.625-0.764)$ and the combined specificity was $95.35 \%$ (95\% CI: $0.815-0.943$ ). The area under the receiver operating characteristic curve (AUC) was 0.857 .

Conclusions: This meta-analysis confirmed that USE shows high sensitivity and specificity in diagnosing benign and malignant thyroid nodules, which could reduce the false negative rate (FNR) and false positive rate (FPR), showing high clinical diagnostic value.

Keywords: Ultrasound elastography (USE); benign and malignant; thyroid nodules

Submitted Jul 12, 2021. Accepted for publication Sep 09, 2021.

doi: $10.21037 / g s-21-492$

View this article at: https://dx.doi.org/10.21037/gs-21-492

\section{Introduction}

Thyroid nodules are one of the most common thyroid diseases in clinical practice, and are caused by a combination of many factors. When thyroid cells proliferate abnormally, a localized mass will appear in the thyroid tissue, and the mass will move up and down with swallowing (1). There are many causes of thyroid nodules, such as inflammation caused by emotional fluctuation, high blood pressure, diabetes, drugs, radiation, and family inheritance (2).

With the improvement of living standards, the public is paying more attention to their own health, and more and more patients are suffering from thyroid nodules. Research literature has shown that the prevalence of thyroid nodules may be related to geographic location and the ethnic distribution of patients. The prevalence of benign and 
malignant thyroid nodules in China can reach up to about $40 \%$. The main distribution feature of the disease is that the prevalence in urban areas is higher than that in rural areas, and the prevalence in coastal areas is higher than that in inland areas (3). Some research reports show that there are significant differences in the prevalence of thyroid nodules among various countries: $20-67 \%$ in the United States, about 30\% in Germany, 27\% in Finland, and 14\% in Ukraine (4).

Conventional ultrasound is the first choice for the diagnosis of thyroid nodules, but it has limitations such as low specificity and crossover of two-dimensional ultrasound images of benign and malignant nodules. Ultrasonic elastography (USE) is a new type of ultrasound imaging technology, first proposed by Ophir et al. in 1991, which mainly focuses on the detection of lesions in the breast, prostate, and blood vessel walls to reflect the biological characteristics of the lesions. In recent years, studies using USE to determine benign and malignant thyroid nodules have gradually increased (5). The prevalence of thyroid nodules in ultrasound screening is $19-67 \%$, of which $5-10 \%$ are malignant nodules (6). USE data can evaluate differences in hardness among different tissues. Many researchers believe that USE shows high sensitivity and specificity in distinguishing benign and malignant thyroid nodules (7).

As a new type of ultrasound imaging technology, USE uses ultra-wide beam tracking technology to obtain the shear wave data of the entire area of interest at the lesion at one time. Through a unique "Shell" analysis tool, it provides various elastic values of the lesion and the periphery of the lesion in real time, so as to acquire twodimensional shear wave elastography (8). USE has obvious advantages in many fields, though the hardness grading standards for different lesions have not been unified (9). Moreover, there are few reports on the application of USE in determining benign and malignant thyroid nodules in the domestic and foreign academic field, and the final diagnosis results are not the same (10).

Meta-analysis was adopted in this study to analyze articles on the identification of benign and malignant thyroid nodules using USE published since the establishment of the utilized databases. This study aims to further explore the application value of USE in the identification of benign and malignant thyroid nodules, and to provide a more objective evidence-based foundation for clinical practice. We present the following article in accordance with the PRISMA reporting checklist (available at https://dx.doi.org/10.21037/ gs-21-492).

\section{Methods}

\section{Article retrieval}

The Chinese Biomedical Literature Database, China National Knowledge Internet (CNKI) Database, Chinese Medical Citation Index (CMCI), Medline, Embase, PubMed, Wanfang Database, VIP Database, and Baidu Scholar were searched to find randomized controlled trials (RCTs) using USE published from the establishment of the database to January 20, 2021. The Boolean logic retrieval method was used to select the relevant articles using compound logic retrieval. Chinese databases were searched with the combination of "ultrasound elastography", "benign and malignant thyroid nodules", and "diagnosis". English databases were searched using "ultrasonic elastography", "benign and malignant thyroid nodules", and "diagnose" as search terms. According to the Rev Man 5.3 software provided by the Cochrane Collaboration, the quality of the included articles was evaluated.

The above 3 search terms were freely combined, and the included articles were determined after multiple searches and then the search engine is used to determine the trace of the documents with the search engine for confirmation. The latest research progress of the studies in the included articles was obtained by contacting experts and researchers in the field.

\section{Inclusion and exclusion criteria}

The inclusion criteria were defined as follows: articles which were RCTs; articles in which patients who were clinically diagnosed with thyroid nodules were the research objects; articles with reliable pathological control analysis and index comparison with the $95 \%$ confidence interval (CI); articles which used USE to diagnose benign and malignant thyroid nodules for patients in the experimental group; and articles including patients whose benign and malignant thyroid nodules met the pathological diagnostic criteria of the Chinese guidelines for malignant risk stratification of thyroid nodules.

The exclusion criteria were as follows: articles which used a special population as the research objects, or with limited age and gender; articles which were repeatedly published; articles which were related to conference speeches, literature reviews, case reports, lectures, etc.; 
articles with unavailable complete data by contacting the original author; and articles which studied patients with non-thyroid nodules.

Two senior experts were invited to screen the topic abstracts and full texts. Three pre-experiments were required before formal screening. If there were inconsistent views between the 2 experts, a consistent conclusion could be obtained through discussion.

\section{Observation indicators}

The included articles were required to analyze the true positive (TP), true negative (TN), false positive (FP), false negative (FN), sensitivity (SEN), and specificity (SPE). The equations of SEN and SPE were as follows:

$$
\begin{aligned}
& \mathrm{SEN}=\mathrm{TP} /(\mathrm{TP}+\mathrm{FN}) \\
& \mathrm{SPE}=\mathrm{TN} /(\mathrm{FP}+\mathrm{TN})
\end{aligned}
$$

\section{Data extraction}

Two experts were invited to extract data independently using a unified Microsoft Excel spreadsheet, and 3 preexperiments were required before extraction. When there were inconsistent views between the 2 experts, a consistent conclusion could be obtained through discussion. The extracted data included: the title of the article; the name of the first author and year of publication; the name of the publication; the research sample size and the type of research design; the general data of research objects; and the evaluation indicators TP, TN, FP, FN, SEN, and SPE.

\section{Assessment of risk bias}

Two experts were invited to assess the risk of bias of the included articles. If they mutually disagreed, the results would be determined through discussion. In this study, the Cochrane Collaboration tool for assessing the risk of bias in RCTs was used. The evaluation criteria included case selection, trials to be evaluated, gold standard, pathological process, and progress. The judgments of "high risk bias", "low risk bias", and "unclear risk bias" were made based on the above 4 aspects.

\section{Quality evaluation}

The quality of the included articles was evaluated by 2 experts. If they mutually disagreed, the results would be determined through discussion. In this study, the Quality
Assessment of Diagnostic Accuracy Studies (QUADAS) tool was used as a quality grading tool for diagnostic tests. If the quality evaluation criteria of the 14 items were all met, the possibility of bias in the study was extremely low (level A). If one or more items were only partially met or not clear, the possibility of bias was medium (level B). If any one or more items were completely inconsistent or incorrect, the article was highly biased (level C). Table 1 shows the specific meanings of the QUADAS tool items.

\section{Statistical analysis}

StataSE12.0 software was applied for statistical analysis. Odds ratio (OR) and mean difference (MD) were used for binary variables and continuous variables, respectively. The bias risk assessment chart was generated by Rev Man5.3 software to evaluate the risk of bias in the included articles. Each effect was represented by a $95 \%$ CI. The SEN and SPE were calculated, the summary receiver operating characteristic (SROC) curve was generated, and the area under the receiver operating characteristic curve (AUC) was calculated. When $\mathrm{P}>0.01$ and $\mathrm{I}^{2}<50 \%$, the fixed effects model (FEM) was used for meta-analysis. When $\mathrm{P}<0.01$ and $\mathrm{I}^{2}>50 \%$, the random effects model (REM) was adopted for meta-analysis.

\section{Results}

\section{Retrieval results and basic information of included articles}

Two hundred and sixty-five literatures were obtained from the database, 169 literatures were obtained from the register, and 65 literatures were excluded in this paper. Forty-three unqualified literatures and 24 literatures for other reasons were excluded, leaving 302 literatures. One hundred and thirty-four literatures were excluded after reading abstract and title, 126 literatures were excluded after reading full text, and 35 literatures were excluded if relevant research information could not be obtained. In addition, 11 literatures were included for meta-analysis after searching through other channels such as web pages (Figure 1). Figure 2 provides the quality evaluation results of the included articles using the QUADAS tool. It revealed that there were 5 level A articles (45.46\%), 4 level B articles (36.36\%), and 2 level C articles (18.18\%).

There were 11 articles that met the inclusion criteria, including 1,411 patients. The 11 articles were all smallsample studies, with sample sizes ranging from 52 to 265 , 
Table 1 Quality Assessment of Diagnostic Accuracy Studies (QUADAS) tool

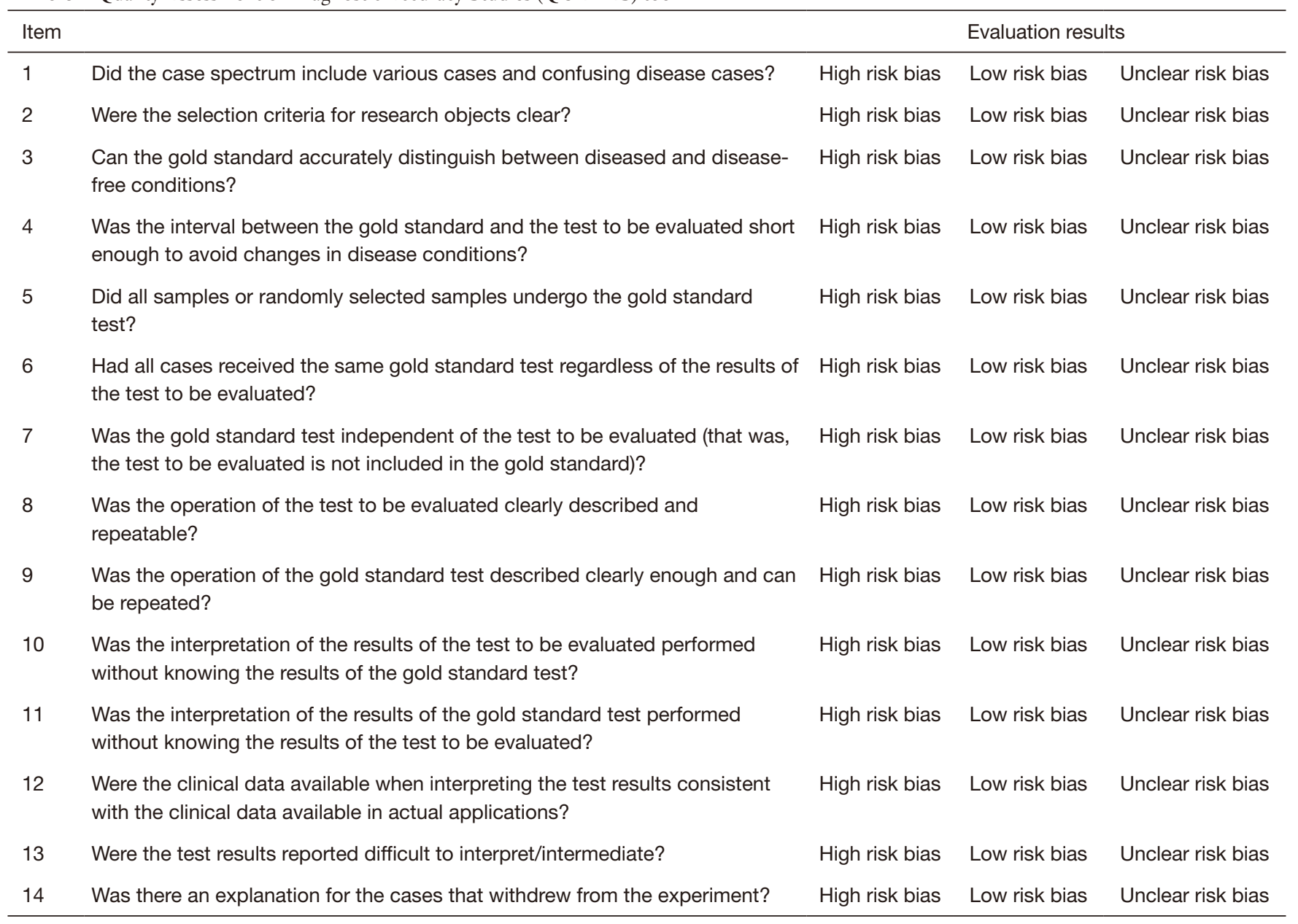

and the age of all research subjects was over 20 years old. The 11 articles described the number of patients, the number of thyroid nodules, TP, TN, FP, and FN in detail. Table 2 shows the basic characteristics of the included articles.

\section{Risk of bias assessment results of the included articles}

Figures 3,4 show the risk of bias assessment results of the included articles, which were generated using Rev Man 5.3 software. Among the 11 RCTs in this study, only 2 articles $(11,12)$ described the correct random allocation method, accounting for $18.18 \%$. Two articles $(13,14)$ described in detail the allocation concealment plan, accounting for $18.18 \%$. One article (15) evaluated the results using the blinding method, accounting for $9.09 \%$. The other articles did not use the blinding method.

\section{Meta-analysis results for the curative effect of clinical symptoms}

A total of 8 articles analyzed the curative effect of clinical symptoms, and the 8 articles used binary variables to describe these curative effects. These studies included 966 patients, with 483 cases in the experimental group and 483 cases in the control group. The overall heterogeneity test was performed, and the results were $\mathrm{Chi}^{2}=7.46, \mathrm{df}=7, \mathrm{I}^{2}=6 \%$, and $\mathrm{P}=0.38$. The research results of Liu BX [2014] (10) accounted for the highest percentage of the final combined results $(31.0 \%)$, followed by the research results of Xue J [2016] (7) (15.2\%). In addition, the horizontal line of the $95 \%$ CI of all included articles was located to the right of the invalid vertical line, so the FEM could be adopted for analysis. The results indicated that the experimental group was significantly different compared with the control group, with $\mathrm{Z}=11.44$, OR =14.58, 95\% CI: 9.21-23.07, and 


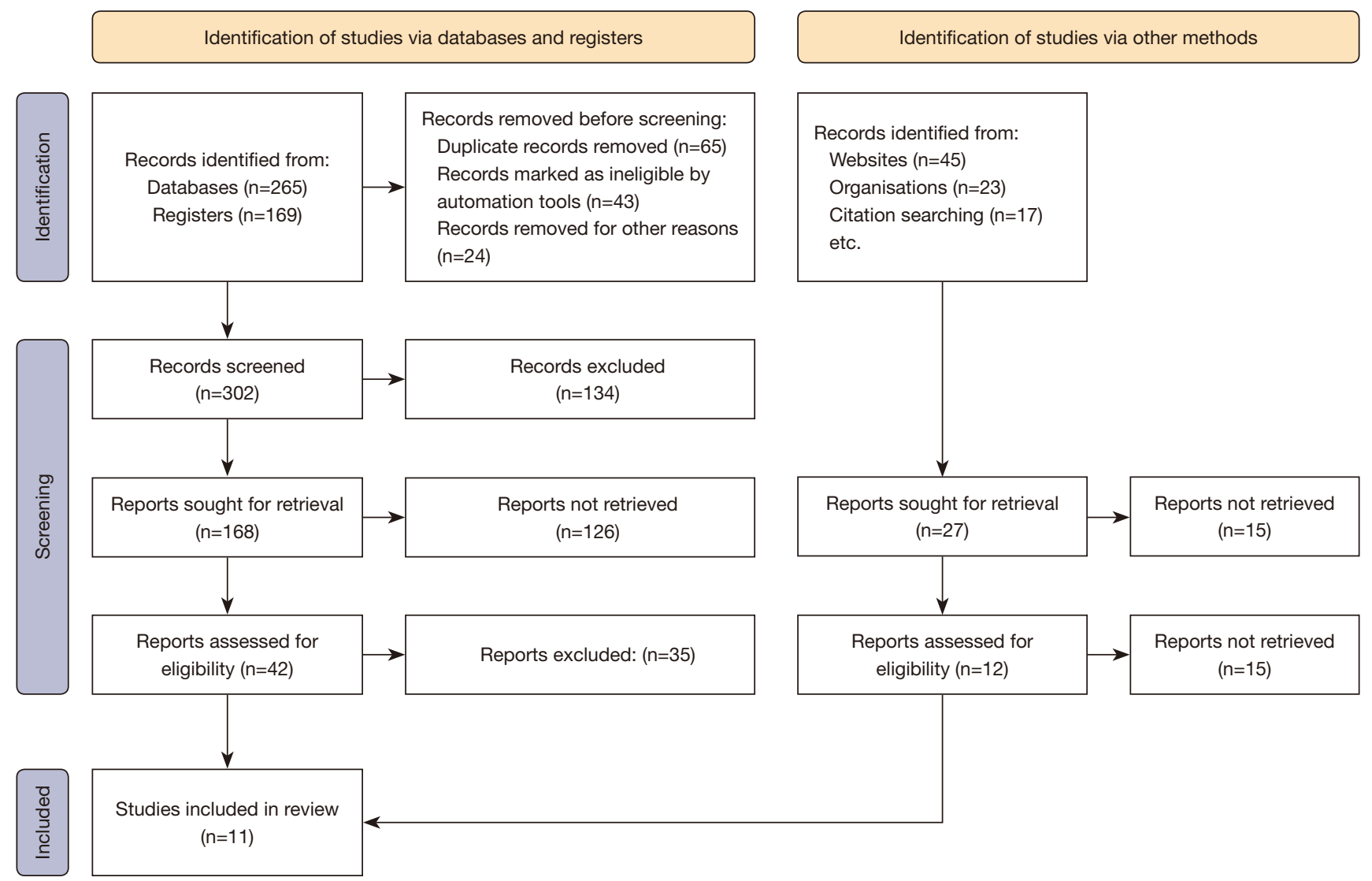

Figure 1 Literature search process.

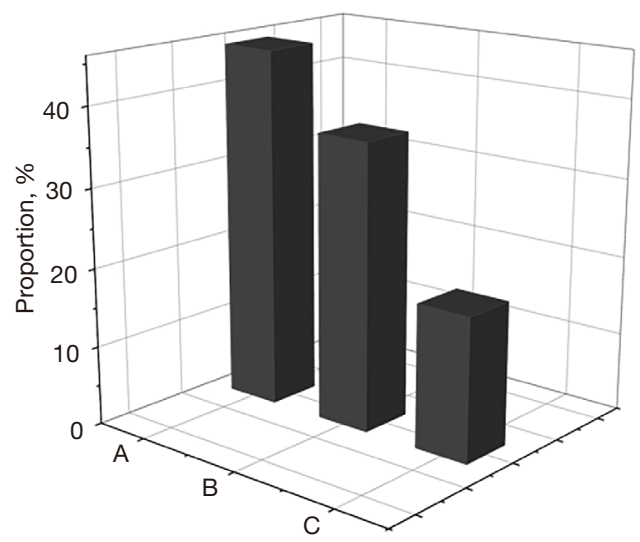

Figure 2 Quality assessment results using the Quality Assessment of Diagnostic Accuracy Studies (QUADAS) tool.

\section{$\mathrm{P}<0.00001$ (as illustrated in Figure 5).}

The funnel plot shown in Figure 6 revealed that the circles of some articles were basically symmetrical with the center line, which indicated that the research accuracy was high, the publication was not biased, and the final conclusions obtained were credible.

\section{Meta-analysis results for the diagnostic efficacy of USE}

A total of 8 articles analyzed the diagnostic efficacy of USE. There were 968 cases in total, including 482 cases in the experimental group, and 486 cases in the control group. The overall heterogeneity test was performed, and the results were $\mathrm{Chi}^{2}=3.03, \mathrm{df}=7, \mathrm{I}^{2}=0 \%$, and $\mathrm{P}=0.88$. The research results of Granchi S [2017] (5) accounted for the highest percentage $(27.0 \%)$ in the final combined results. The horizontal line of the $95 \%$ CI of the 6 articles was located to the right of the invalid vertical line. The analysis results of FEM (Figure 7) suggested that the experimental group was significantly different compared to the control group, with $Z=6.33$, OR $=7.47,95 \% \mathrm{CI}$ : 4.01-13.93), and $\mathrm{P}<0.00001$.

The funnel plot (Figure 8) showed that the circles of the included articles were basically symmetrical with the 
Table 2 The basic characteristics of the included articles

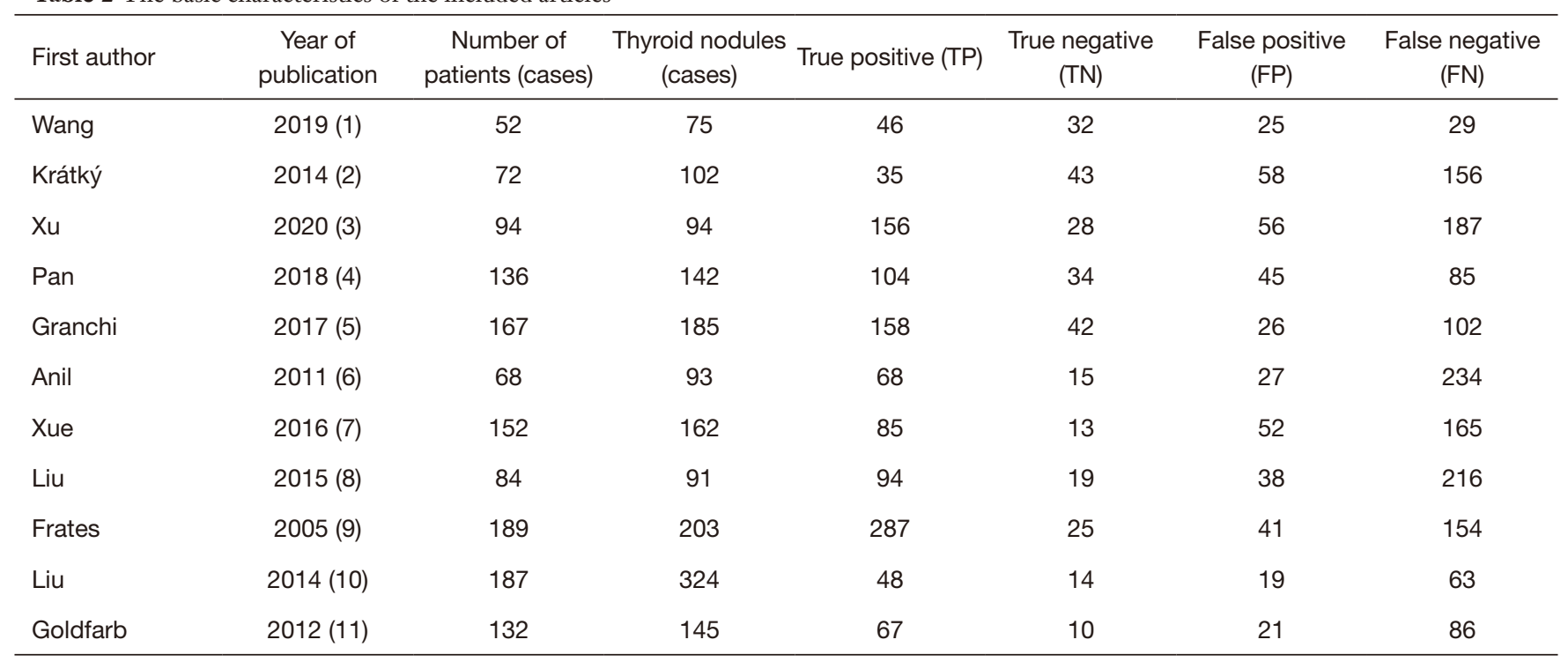

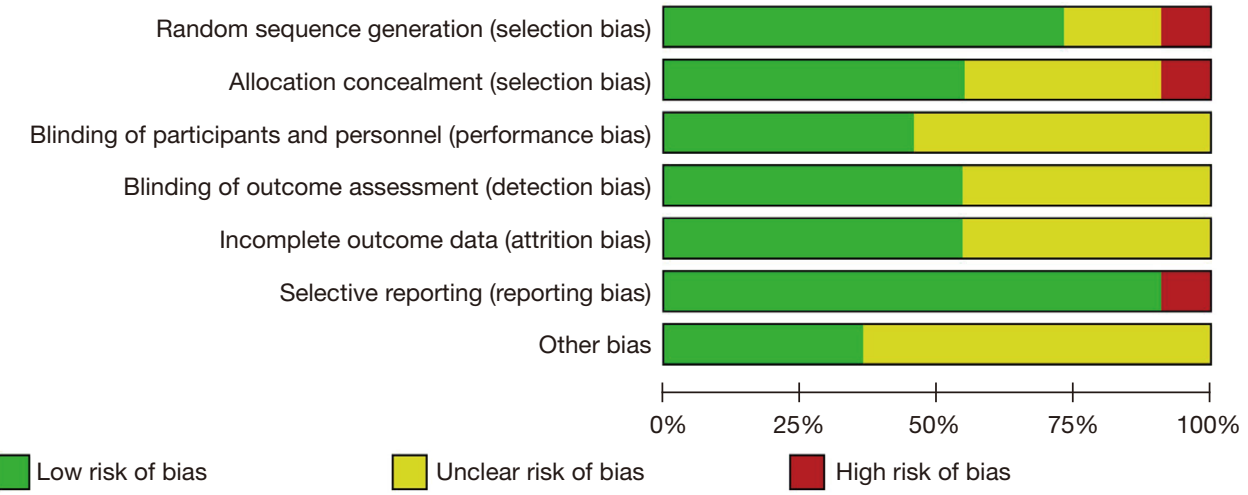

Figure 3 Bar graph showing the bias assessment of the included articles.

midline, which indicated that the research accuracy was high, the publication was not biased, and the final conclusions obtained were credible.

\section{Meta-analysis of pathological diagnosis results}

A total of 10 literatures analyzed the pathological diagnosis results in randomized controlled trials. There were 1,201 cases in total, with 602 cases in the experimental group and 599 cases in the control group. The overall heterogeneity test showed that $\mathrm{Tau}^{2}=0.78, \mathrm{Chi}^{2}=20.97, \mathrm{df}=9, \mathrm{I}^{2}=57 \%$, and $\mathrm{P}=0.01$. The research results of Liu B [2015] (8) accounted for the highest percentage of the final combined results $(10.8 \%)$. The horizontal line of the $95 \%$ CI of the 10 articles was to the right of the invalid vertical line. The analysis results of REM revealed that the difference between the experimental group and the control group was statistically significant, with $Z=7.27, \mathrm{OR}=14.67,95 \% \mathrm{CI}$ : 7.11-30.27), and $\mathrm{P}<0.00001$ (as illustrated in Figure 9).

The funnel plot (Figure 10) showed that the circles of the included articles were basically symmetrical with the midline, which indicated that the research accuracy was high, the publication was not biased, and the final conclusions obtained were credible.

\section{Meta-analysis results of combined SEN and combined SPE}

After meta-analysis, the combined SEN was $72.26 \%$ (95\% 


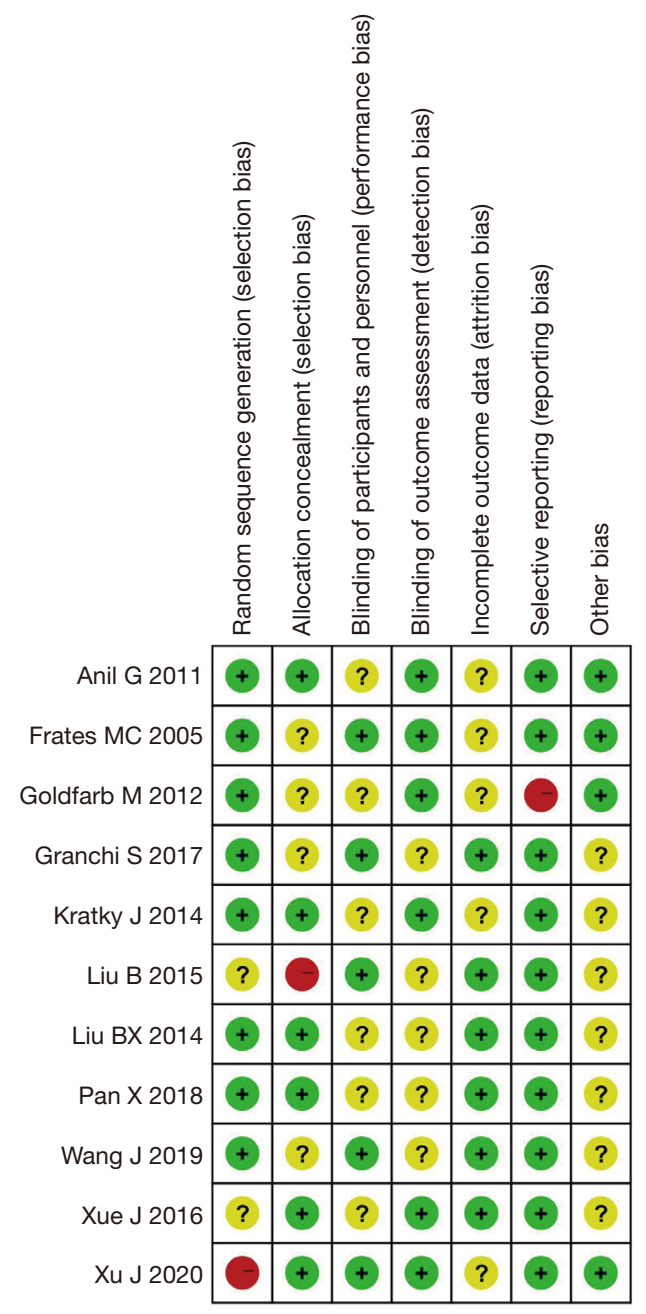

Figure 4 Results of the bias assessment of included articles.
CI: $0.625-0.764)$ and the combined SPE was $95.35 \%$ (95\% CI: 0.815-0.943). The SROC showed that the AUC was 0.857 (Figure 11).

\section{Discussion}

In this study, meta-analysis was adopted to analyze the identification of benign and malignant thyroid nodules using USE. The internal structure of the thyroid nodule tissue changes, and the hardness of the lesion tissue also changes. The hardness classification of USE is based on changes in the pathological structure of the thyroid tissue, which can reflect the different elasticity of the lesion tissue (16).

In this study, 11 randomized controlled trials were included, including 1,333 patients with thyroid nodules. A total of 1,616 thyroid nodules were detected, indicating single or multiple nodules. Only 2 randomized controls (18.18\%) described the correct method of randomization. Two articles (18.18\%) describe the distribution scheme concealment in detail. Results were evaluated by blind method in 1 article $(9.09 \%)$, and not in the rest. By comparing the ultrasound elastography diagnosis of benign and malignant thyroid nodules with the biopsy pathological diagnosis results, it was found that the included study had high accuracy, there was no bias in publication, and the final conclusion was relatively credible $(17,18)$.

Internationally, the TP, FP, TN, and $\mathrm{FN}$ values of statistical diagnostic methods are the standard methods to determine the accuracy of the test. Therefore,

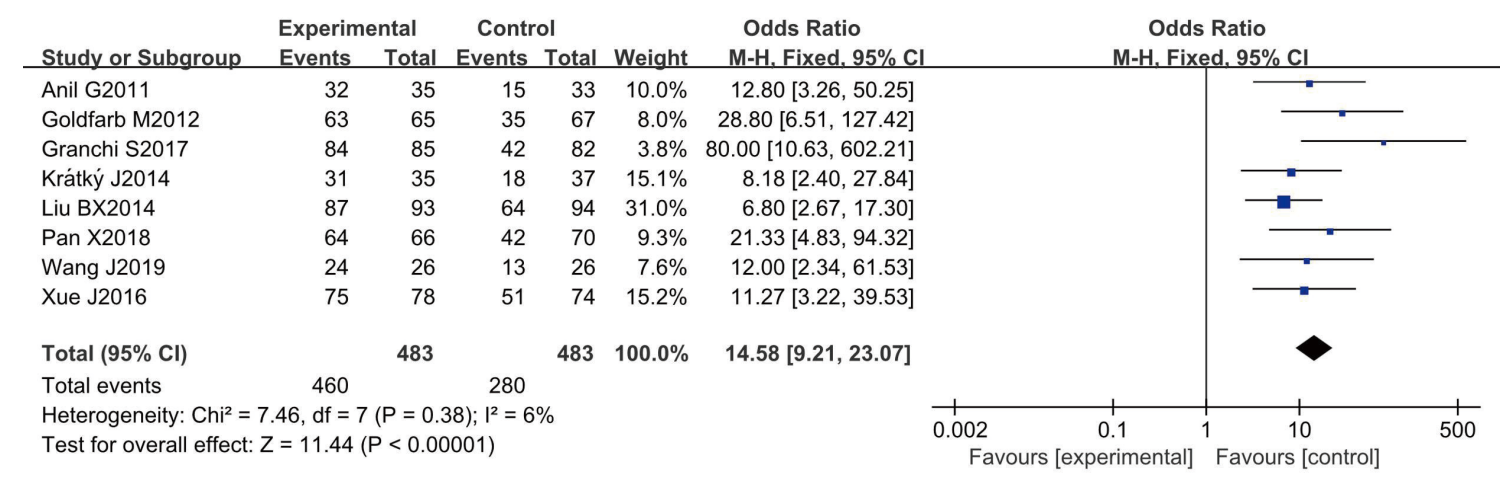

Figure 5 Forest plot of the fixed effects model (FEM) results of the curative effect of clinical symptoms. 


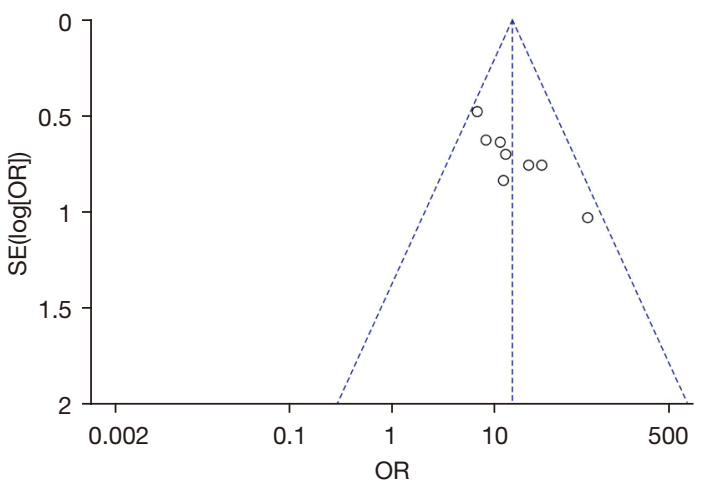

Figure 6 Funnel plot of the fixed effects model (FEM) results of the curative effect of clinical symptoms.

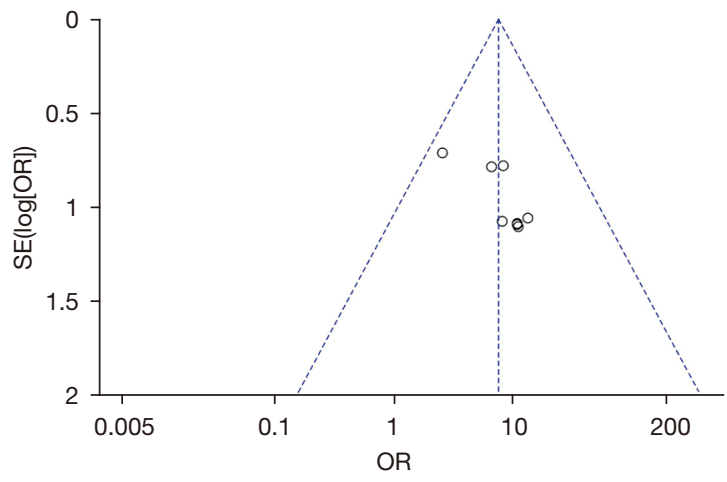

Figure 8 Funnel plot of the fixed effects model (FEM) results of the diagnostic efficacy of ultrasonic elastography (USE).

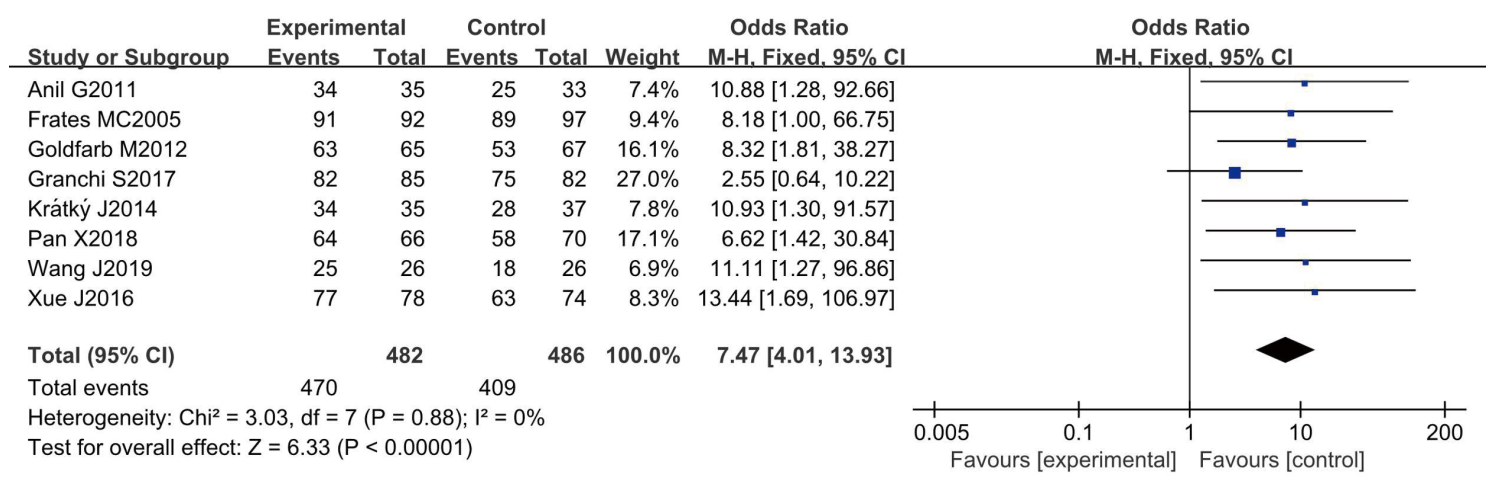

Figure 7 Forest plot of the fixed effects model (FEM) results for the diagnostic efficacy of ultrasonic elastography (USE).

calculating the SEN and SPE of diagnostic methods can comprehensively evaluate the application value of USE as a diagnostic method (19). In the 11 included articles in this study, the TP, FP, TN, and FN values were described in detail. Two articles calculated the diagnostic coincidence rate and compared the processes of USE diagnosis and biopsy pathological diagnosis in patients with thyroid nodules (20). However, it is necessary to obtain more relevant data to make the diagnostic results more accurate (21).

Eight included articles analyzed the diagnostic efficacy of USE. There were 968 cases in total, including 482 cases in the experimental group and 486 cases in the control group. The overall heterogeneity test was performed, and the results were $\mathrm{Chi}^{2}=3.03, \mathrm{df}=7, \mathrm{I}^{2}=0 \%$, and $\mathrm{P}=0.88$ (22), therefore, the FEM was adopted for analysis. The results revealed that the experimental group was significantly different compared with the control group, with $\mathrm{Z}=6.33$, $\mathrm{OR}=7.47,95 \%$ CI: 4.01-13.93, and $\mathrm{P}<0.01$.
The SROC curve allows for the visual comparison of diagnostic tests through graphs and areas, avoiding the shortcomings of previous meta-analysis methods. AUC $<0.5$ indicates that there is no diagnostic value, $0.5<\mathrm{AUC}$ $<0.7$ indicates diagnostic value, $0.7<\mathrm{AUC}<0.9$, indicates that the diagnostic value is high, and $\mathrm{AUC}=0.9$ indicates that the diagnostic value is very high (23). In this study, the meta-analysis results suggested that the combined SEN was $72.26 \%$ (95\% CI: $0.625-0.764$ ), the false negative rate (FNR) was $12.5 \%$, the combined SPE was $95.35 \%(95 \%$ CI: $0.815-0.943$ ), and the false positive rate (FPR) was $10.3 \%$. The SROC curve showed that the AUC was 0.857 , indicating that USE has high diagnostic value for benign and malignant thyroid nodules and could reduce the FNR and FPR, which was consistent with the results of Sun et al. [2014] (24).

The QUADAS tool was adopted to evaluate the quality of the included articles. The results showed that there were 5 level A articles (45.46\%), 4 level B articles (36.36\%), 


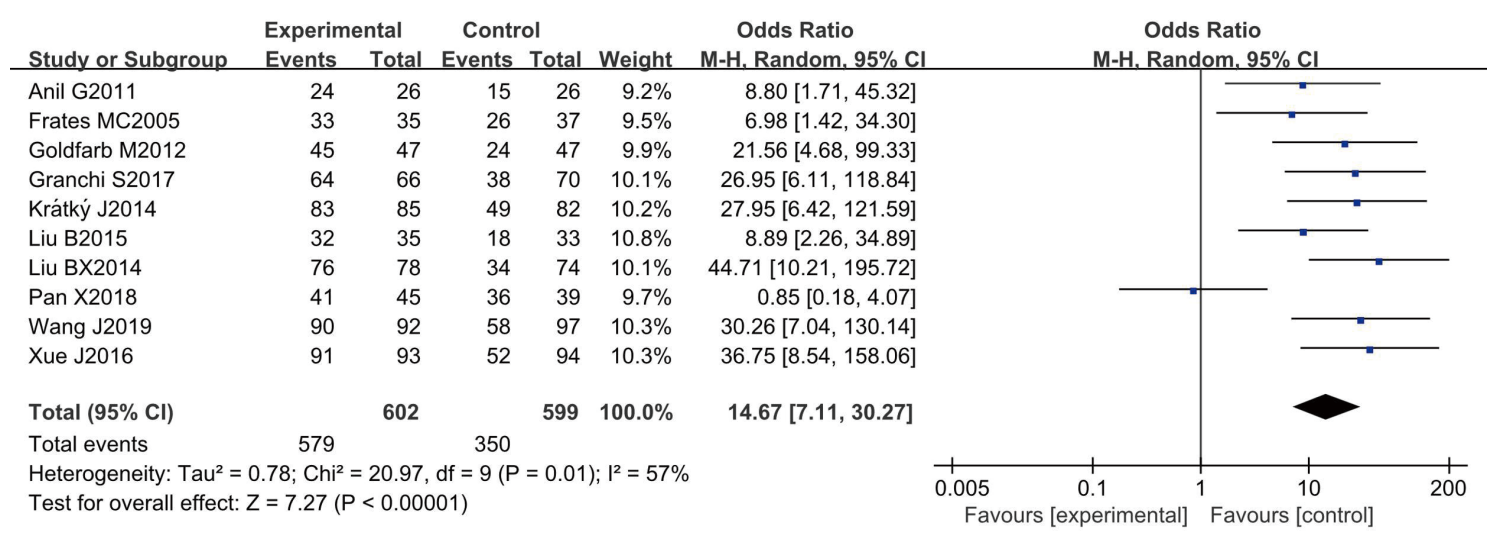

Figure 9 Forest plot of the random effects model (REM) results of the therapeutic effect of pathological diagnosis.

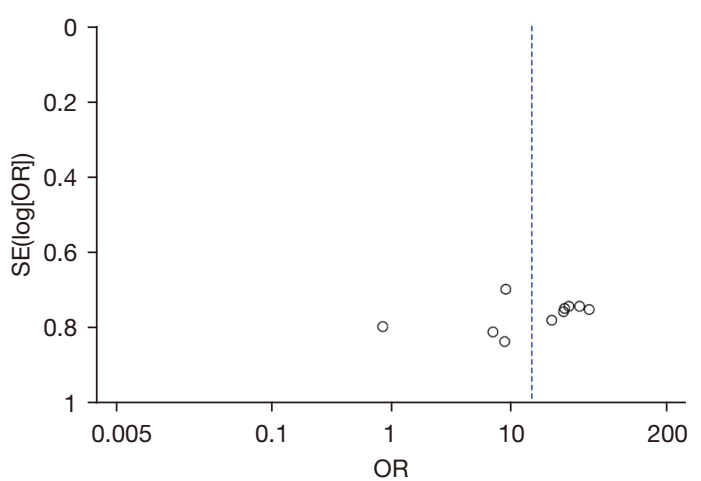

Figure 10 Funnel plot of the random effects model (REM) results of the therapeutic effect of pathological diagnosis.

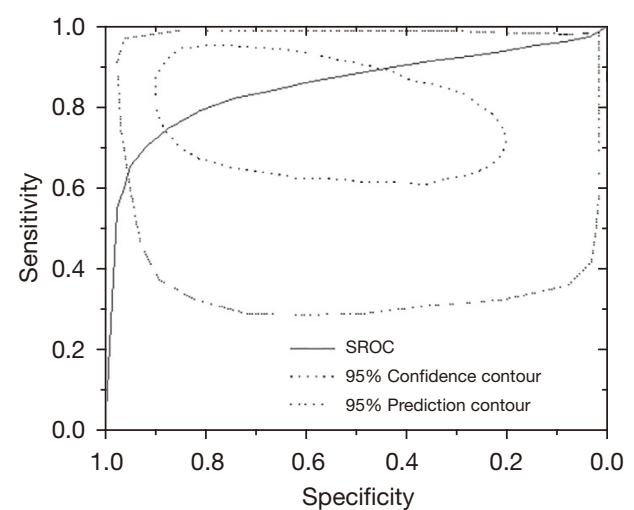

Figure 11 Summary receiver operating characteristic (SROC) curve.

and 2 level C articles (18.18\%) (25). However, the sample size of the research included in this meta-analysis was relatively small, the quality of the research was not high enough, and the quality level of the included experiments was average.

\section{Conclusions}

In this study, the Boolean logic search method was used to identify 11 articles which used USE to determine benign and malignant thyroid nodules. Biopsy pathological diagnosis was undertaken as the gold standard for the metaanalysis to evaluate the diagnostic value of USE for the identification of benign and malignant thyroid nodules. The meta-analysis of this study confirmed that USE showed high SEN and SPE in diagnosing benign and malignant thyroid nodules, could reduce the FNR and FPR, and showed high clinical diagnostic value. The shortcomings of this study were that the sample size was small and unpublished literature was not obtained, so articles with certain potential bias could not be excluded. Therefore, the conclusions of this study still require further verification by high-quality research. Overall, USE can be used to diagnose thyroid nodules for patients who are not suitable for or do not accept biopsy diagnosis.

\section{Acknowledgments}

Funding: None.

\section{Footnote}

Reporting Checklist: The authors have completed the PRISMA reporting checklist. Available at https://dx.doi. org/10.21037/gs-21-492

Conflicts of Interest: All authors have completed the ICMJE uniform disclosure form (available at https://dx.doi. org/10.21037/gs-21-492). The authors have no conflicts of interest to declare. 
Ethical Statement: The authors are accountable for all aspects of the work in ensuring that questions related to the accuracy or integrity of any part of the work are appropriately investigated and resolved.

Open Access Statement: This is an Open Access article distributed in accordance with the Creative Commons Attribution-NonCommercial-NoDerivs 4.0 International License (CC BY-NC-ND 4.0), which permits the noncommercial replication and distribution of the article with the strict proviso that no changes or edits are made and the original work is properly cited (including links to both the formal publication through the relevant DOI and the license). See: https://creativecommons.org/licenses/by-nc-nd/4.0/.

\section{References}

1. Wang J, Wei W, Guo R. Ultrasonic elastography and conventional ultrasound in the diagnosis of thyroid micronodules. Pak J Med Sci 2019;35:1526-31.

2. Krátký J, Vítková H, Bartáková J, et al. Thyroid nodules: pathophysiological insight on oncogenesis and novel diagnostic techniques. Physiol Res 2014;63 Suppl 2:S263-75.

3. Xu J, Wang P, Yue W, et al. Application of ultrasonic shear wave elastography and contrast-enhanced ultrasound in the differential diagnosis of patients with benign and malignant thyroid lesions. Gland Surg 2020;9:2136-43.

4. Pan X, Wang L. Comparison of diagnostic values between ultrasound elastography and ultrasound-guided thyroid nodular puncture in thyroid nodules. Oncol Lett 2018;16:5209-13.

5. Granchi S, Vannacci E, Biagi E. Characterization of benign thyroid nodules with HyperSPACE (Hyper Spectral Analysis for Characterization in Echography) before and after percutaneous laser ablation: a pilot study. Med Ultrason 2017;19:172-8.

6. Anil G, Hegde A, Chong FH. Thyroid nodules: risk stratification for malignancy with ultrasound and guided biopsy. Cancer Imaging 2011;11:209-23.

7. Xue J, Cao XL, Shi L, et al. The diagnostic value of combination of TI-RADS and ultrasound elastography in the differentiation of benign and malignant thyroid nodules. Clin Imaging 2016;40:913-6.

8. Liu B, Liang J, Zheng Y, et al. Two-dimensional shear wave elastography as promising diagnostic tool for predicting malignant thyroid nodules: a prospective singlecentre experience. Eur Radiol 2015;25:624-34.
9. Frates MC, Benson CB, Charboneau JW, et al. Management of thyroid nodules detected at US: Society of Radiologists in Ultrasound consensus conference statement. Radiology 2005;237:794-800.

10. Liu BX, Xie XY, Liang JY, et al. Shear wave elastography versus real-time elastography on evaluation thyroid nodules: a preliminary study. Eur J Radiol 2014;83:1135-43.

11. Goldfarb M, Gondek SS, Sanchez Y, et al. Clinic-based ultrasound can predict malignancy in pediatric thyroid nodules. Thyroid 2012;22:827-31.

12. Liu MJ, Men YM, Zhang YL, et al. Improvement of diagnostic efficiency in distinguishing the benign and malignant thyroid nodules via conventional ultrasound combined with ultrasound contrast and elastography. Oncol Lett 2017;14:867-71.

13. Huang S, Meng N, Pan M, et al. Diagnostic performances of the KWAK-TIRADS classification, elasticity score, and Bethesda System for Reporting Thyroid Cytopathology of TI-RADS category 4 thyroid nodules. Int J Clin Exp Pathol 2020;13:1159-68.

14. Liu B, Liang J, Zhou L, et al. Shear Wave Elastography in the Diagnosis of Thyroid Nodules with Coexistent Chronic Autoimmune Hashimoto's Thyroiditis. Otolaryngol Head Neck Surg 2015;153:779-85.

15. Yang J, Song Y, Wei W, et al. Comparison of the effectiveness of ultrasound elastography with that of conventional ultrasound for differential diagnosis of thyroid lesions with suspicious ultrasound features. Oncol Lett 2017;14:3515-21.

16. Gao LY, Wang Y, Jiang YX, et al. Ultrasound is helpful to differentiate Bethesda class III thyroid nodules: A PRISMA-compliant systematic review and meta-analysis. Medicine (Baltimore) 2017;96:e6564.

17. Tessler FN, Middleton WD, Grant EG, et al. ACR Thyroid Imaging, Reporting and Data System (TI-RADS): White Paper of the ACR TI-RADS Committee. J Am Coll Radiol 2017;14:587-95.

18. Lee JY, Na DG, Yoon SJ, et al. Ultrasound malignancy risk stratification of thyroid nodules based on the degree of hypoechogenicity and echotexture. Eur Radiol 2020;30:1653-63.

19. Singaporewalla RM, Hwee J, Lang TU, et al. Clinicopathological Correlation of Thyroid Nodule Ultrasound and Cytology Using the TIRADS and Bethesda Classifications. World J Surg 2017;41:1807-11.

20. Shin JH, Baek JH, Chung J, et al. Ultrasonography Diagnosis and Imaging-Based Management of Thyroid 
Nodules: Revised Korean Society of Thyroid Radiology

Consensus Statement and Recommendations. Korean J

Radiol 2016;17:370-95.

21. Prochazka A, Gulati S, Holinka S, et al. Classification of Thyroid Nodules in Ultrasound Images Using DirectionIndependent Features Extracted by Two-Threshold Binary Decomposition. Technol Cancer Res Treat 2019; 18:1533033819830748.

22. Gao L, Xi X, Wang J, et al. Ultrasound risk evaluation of thyroid nodules that are "unspecified" in the 2015 American Thyroid Association management guidelines: A retrospective study. Medicine (Baltimore) 2018;97:e13914.

23. Moon WJ, Baek JH, Jung SL, et al. Ultrasonography and

Cite this article as: Zhou Y, Chen H, Qiang J, Wang D, Systematic review and meta-analysis of ultrasonic elastography in the diagnosis of benign and malignant thyroid nodules. Gland Surg 2021;10(9):2734-2744. doi: 10.21037/gs-21-492 the ultrasound-based management of thyroid nodules: consensus statement and recommendations. Korean J Radiol 2011;12:1-14.

24. Sun J, Cai J, Wang X. Real-time ultrasound elastography for differentiation of benign and malignant thyroid nodules: a meta-analysis. J Ultrasound Med 2014;33:495-502.

25. Mihailescu DV, Collins BJ, Wilbur A, et al. Ultrasounddetected thyroid nodules in radiation-exposed patients: changes over time. Thyroid 2005;15:127-33.

(English Language Editor: C. Betlazar-Maseh) 